\title{
The Conserved Arginine Required for AvrRps4 Processing Is Also Required for Recognition of Its $\mathrm{N}$-Terminal Fragment in Lettuce
}

\author{
Jianbin Su, ${ }^{1,2}$ Quang-Minh Nguyen, ${ }^{3}$ Ashten Kimble, ${ }^{2,4}$ Sharon M. Pike, ${ }^{1,2}$ Sang Hee Kim, ${ }^{3}$ and \\ Walter Gassmann ${ }^{1,2,+}$ \\ ${ }^{1}$ Division of Plant Sciences, University of Missouri, Columbia, MO 65211, U.S.A. \\ ${ }^{2}$ Christopher S. Bond Life Sciences Center and Interdisciplinary Plant Group, University of Missouri, Columbia, MO 66211, \\ U.S.A. \\ ${ }^{3}$ Division of Applied Life Science (BK21 Plus), Plant Molecular Biology and Biotechnology Research Center, Gyeongsang \\ National University, Jinju 52828, Korea \\ ${ }^{4}$ Division of Biological Sciences, University of Columbia, MO 65211, U.S.A.
}

Accepted 4 November 2020.

\begin{abstract}
Pathogens utilize a repertoire of effectors to facilitate pathogenesis, but when the host recognizes one of them, it causes effector-triggered immunity. The Pseudomonas type III effector AvrRps4 is a bipartite effector that is processed in planta into a functional 133-amino acid N-terminus (AvrRps4-N) and 88-amino acid C-terminus (AvrRps4-C). Previous studies found AvrRps4-C to be sufficient to trigger the hypersensitive response (HR) in turnip. In contrast, our recent work found that AvrRps4-N but not AvrRps4-C triggered HR in lettuce, whereas both were required for resistance induction in Arabidopsis. Here, we initially compared AvrRps4 recognition by turnip and lettuce using transient expression. By serial truncation, we identified the central conserved region consisting of 37 amino acids as essential for AvrRps4-N recognition, whereas the putative type III secretion signal peptide or the C-terminal 13 amino acids were dispensable. Surprisingly, the conserved arginine at position 112 (R112) that is required for full-length AvrRps4 processing is also required for the recognition of AvrRps4-N by lettuce. Mutating R112 to hydrophobic leucine or negatively charged glutamate abolished the HR-inducing capacity of AvrRps4-N, while a positively charged lysine at this position resulted in a slow and weak HR. Together, our results suggest an AvrRps4-N recognition-specific role of R112 in lettuce.
\end{abstract}

Keywords: AvrRps4, effector-triggered immunity (ETI), conserved arginine, lettuce, turnip

Current affiliation for Ashten Kimble: Bayer Crop Science, Chesterfield MO 63107, U.S.A.

${ }^{\dagger}$ Corresponding author: W. Gassmann: gassmannw@missouri.edu

Funding: This research was funded in part by National Science Foundation grant IOS-1456181 (W. Gassmann), and by a grant from the NextGeneration BioGreen 21 Program, Rural Development Administration, Republic of Korea (SSAC, grant PJ01344901) (S. H. Kim).

*The $\boldsymbol{e}$-Xtra logo stands for "electronic extra" and indicates there is supplementary material published online.

The author(s) declare no conflict of interest.

(c) (1) () $\odot$ Copyright $\odot 2021$ The Author(s). This is an open access article distributed under the CC BY-NC-ND 4.0 International license.
The plant immune system has developed a two-layered defense (Jones and Dangl 2006). In the first layer, plasma membrane-localized receptors detect the presence of conserved microbial molecular patterns, known as microbial/pathogen associated molecular patterns (MAMPs/PAMPs), to initiate MAMP/ PAMP-triggered immunity (MTI/PTI). To overcome host MTI/ PTI, a sophisticated pathogen delivers a repertoire of virulence effectors into plant cells to interfere with plant functions at multiple levels (Block and Alfano 2011; Feng and Zhou 2012; Jones and Dangl 2006; Su et al. 2018; Xin and He 2013). A second layer of the plant immune system utilizes resistance (R) proteins to interact with effectors directly or to monitor effector-induced modifications. Recognition of effectors by intracellular $\mathrm{R}$ proteins initiates effector-triggered immunity (ETI), which is often associated with rapid cell death known as the hypersensitive response (HR) (Cui et al. 2015; Jones and Dangl 2006; Kourelis and van der Hoorn 2018; Lolle et al. 2020; Spoel and Dong 2012; Su et al. 2018).

In Arabidopsis, it is established that two $\mathrm{R}$ protein pairs, RRS1-RPS4 and RRS1B-RPS4B, function redundantly to recognize the Pseudomonas type III effector AvrRps4 (Gassmann et al. 1999; Hinsch and Staskawicz 1996; Narusaka et al. 2009; Saucet et al. 2015). AvrRps4 was shown to target the WRKY domain of RRS1/RRS1B (Ma et al. 2018; Sarris et al. 2015). This interaction was proposed to disrupt RRS1/RRS1B intramolecular WRKY domain association with an adjacent domain, which activates immune signaling (Ma et al. 2018; Sarris et al. 2015). Besides its interaction with the WRKY domains of RRS1 and RRS1B, AvrRps4 also coimmunoprecipitates with some key immune-responsive WRKY transcription factors, including WRKY33, WRKY41, WRKY60, and WRKY70 (Sarris et al. 2015). Thus, the virulence function of AvrRps4 may consist of dampening the immune output by interfering with WRKY transcription factors.

AvrRps4 was previously shown to be processed into a 133amino acid N-terminus (AvrRps4-N) and an 88-amino acid Cterminus (AvrRps4-C) in planta and the AvrRps4-N amino acid R112 was critical for processing (Sohn et al. 2009). Estradiolinducible expression of AvrRps4-C alone in the Arabidopsis accession Columbia-0 (Col-0) triggers HR to a similar extent as full-length AvrRps4 (Li et al. 2014), which correlates well with the finding that transient overexpression of AvrRps4-C is sufficient to trigger HR in turnip (Sohn et al. 2009). In contrast, 
dexamethasone (DEX)-inducible expression of AvrRps4-N in Col-0 alone does not trigger HR (Halane et al. 2018). It is worth noting that the Pseudomonas type III secretion system-mediated delivery of AvrRps4 at natural levels does not trigger HR in Col-0, though it triggers strong resistance to the Pseudomonas syringae pv. tomato strain DC3000 (DC3000) (Gassmann 2005). Thus, HR caused by estradiol-inducible expression of AvrRps4-C or full-length AvrRps4 in Col-0 might be an artificial effect resulting from transient overexpression, a factor that should be taken into consideration when interpreting AvrRps4 avirulence results. Nonetheless, transient overexpressionmediated HR has proven to be a rapid and useful tool to study RPS4-RRS1- and RPS4B-RRS1B-mediated recognition of AvrRps4 (Ma et al. 2018; Sarris et al. 2015; Saucet et al. 2015).

We recently demonstrated that AvrRps4-N and AvrRps4-C are mutually required to trigger resistance in Arabidopsis when delivered by the Pseudomonas type III secretion system, either as a full-length protein or when delivered by separate constructs (Halane et al. 2018). Previously, it was proposed that AvrRps4$\mathrm{N}$ only contains a type III secretion and a chloroplast-targeting signal and that AvrRps4-C alone is responsible for triggering resistance by targeting RPS4-RRS1 and RPS4B-RRS1B R protein pairs (Ma et al. 2018; Li et al. 2014; Sarris et al. 2015; Saucet et al. 2015; Sohn et al. 2009). However, findings that AvrRps4 also interacts with the RPS4-RRS1-associated immune regulator EDS1 (Bhattacharjee et al. 2011; Heidrich et al. 2011) and that this interaction is predominantly with AvrRps4$\mathrm{N}$ (Halane et al. 2018) indicate that details of AvrRps4-N recognition and its coordination with AvrRps4-C recognition in planta to trigger resistance remain to be determined and are more complicated than previously thought.

Our proposition that AvrRps4-N retains effector function is consistent with the observation that AvrRps4-N promotes bacterial virulence when overexpressed in Col-0 (Halane et al. 2018). This virulence function of AvrRps4-N is supported by an earlier study, in which AvrRps4-mediated suppression of flg22-induced reactive oxygen species burst and callose deposition required AvrRps4-N (Li et al. 2014). The conservation of amino acid sequences in the processed N-termini of the Pseudomonas effectors AvrRps4 and HopK1 and the Xanthomonas effector XopO (Li et al. 2014) provides additional support for a conserved function that extends beyond serving as a type III secretion signal. Based on the distinct virulence and avirulence functions of AvrRps4-N and AvrRps4-C, AvrRps4 was proposed to be a bipartite effector (Halane et al. 2018). The mosaic nature of AvrRps4 is shared with XopO and HopK1; while XopO has a C-terminal domain with sequence similarity to AvrRps4-C, the HopK1 C-terminal sequence is unrelated to AvrRps4-C (Halane et al. 2018; Li et al. 2014).

As AvrRps4-N alone does not induce any drastic HR phenotype in Arabidopsis, it is difficult to study effector functions in that system. Fortunately, we identified an HR-inducing activity of AvrRps4-N in lettuce (Halane et al. 2018), which opens opportunities for molecular studies of AvrRps4-N effector functions and recognition mechanisms. Notably, there are no obvious RRS1 orthologs or similar TNL-WRKY domain fusions in the lettuce genome (Reyes-Chin-Wo et al. 2017). The absence of an appropriate AvrRps4-C target may explain why its expression does not trigger $\mathrm{HR}$ in lettuce and instead dampens the HR induced by AvrRps4-N (Halane et al. 2018). The opposite results in turnip and lettuce indicate that AvrRps4 evolved from the terminal reassortment of two independent effector domains (Halane et al. 2018; Stavrinides et al. 2006).

In the present study, we initially compared turnip and lettuce responses to AvrRps4 to gain insights into structural features of AvrRps4 required for recognition in lettuce. Through truncations and point mutations, we found that the central conserved middle region and a conserved arginine at position 112 are required for AvrRps4-N recognition in lettuce.

\section{RESULTS}

Differential recognition of AvrRps4 by lettuce and turnip.

We first compared AvrRps4 recognition in lettuce (cv. Kordaat) and turnip (cv. PI227296) in detail by transiently expressing hemagglutinin (HA)-tagged versions of AvrRps4, namely, HAAvrRps4-N, HA-AvrRps4-C, and HA-AvrRps4-FL. Consistent with previous reports (Sohn et al. 2009; Halane et al. 2018), HAAvrRps4-N and HA-AvrRps4-FL but not HA-AvrRps4-C trigger strong HR in lettuce, while in turnip HA-AvrRps4-C and HAAvrRps4-FL but not HA-AvrRps4-N induce HR (Fig. 1A and C).

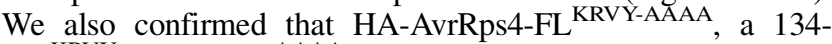
$137^{\mathrm{KRVY}}$ to $134-137^{\mathrm{AAAA}}$ mutant, was unable to cause HR in turnip (Fig. 1C), as previously reported (Sohn et al. 2009). This mutant can still trigger HR in lettuce (Fig. 1A). Because HAAvrRps4-FL ${ }^{\text {KRVY-AAAA }}$ can be processed to generate functional HA-AvrRps4-N (Sohn et al. 2009) (Fig. 1B), it is reasonable that

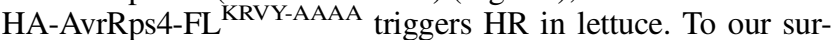
prise, HA-AvrRps4-FL ${ }^{\mathrm{R} 112 \mathrm{~L}}$, a processing-deficient mutant that maintains the ability to cause HR in turnip (Sohn et al. 2009) (Fig. 1B and C), fails to induce HR in lettuce (Fig. 1A). These results indicate that, for lettuce $H R$, these mutations in the AvrRps4 C-terminus but not in the N-terminus are tolerated, whereas the opposite is true for turnip.

\section{Ninja and Hilde fail to recognize AvrRps4-N.}

When we assayed HR in turnip, we found that PI212593, another turnip cultivar, fails to recognize AvrRps4-FL and AvrRps4-C. Because of less efficient transient expression in turnip, protein accumulated at very low levels. Nevertheless, we could show that AvrRps4 expression in PI212593 is higher than in PI227296 (Supplementary Fig. S1), suggesting that the absence of HR in PI212593 is not caused by insufficient transient protein expression but is most likely because of an absence of the corresponding $R$ gene or genes. This led us to screen whether there are other lettuce cultivars that do not respond to AvrRps4-N. AvrRps4-N and an empty vector were infiltrated into six lettuce cultivars and five lines of wild lettuce (Lactuca serriola), the progenitor of modern lettuce. Salad Bowl and Salinas-88 produced HR similar to that in Kordaat, Valmaine produced weaker HR, while Ninja and Hilde failed to recognize AvrRps4-N (Fig. 2A), which makes it possible to map the corresponding $R$ gene or genes. AvrRps4-N expression levels were comparable among these cultivars, as shown by Western blot (Fig. 2B). In this limited survey of lettuce cultivars, the HR pattern induced by AvrRps4-N is identical to that induced by AvrRps4-FL (Wroblewski et al. 2009). Interestingly, among the five tested wild lettuce collections, two of them recognized AvrRps4-N, while the other three did not despite comparable AvrRps4-N protein levels (Fig. 2C and D). This suggests that the genetics of AvrRps4-N recognition are polymorphic in wild lettuce, which likely forms the basis of recognition presence/absence in lettuce cultivars.

\section{R112 is required for AvrRps4-N recognition in lettuce.}

The result with the R112L mutation in AvrRps4-FL raised the question whether R112-mediated AvrRps4 cleavage or a processing-independent mechanism mediates AvrRps4-N recognition. To test these possibilities, we examined whether the R112L mutation in AvrRps4-N (AvrRps4-N ${ }^{\mathrm{R} 112 \mathrm{~L}}$ ) can trigger $\mathrm{HR}$ in Kordaat. As is shown, similar to AvrRps4-FL ${ }^{\mathrm{R} 12 \mathrm{~L}}$, AvrRps4-N ${ }^{\mathrm{R} 112 \mathrm{~L}}$ fails to trigger HR (Fig. 3A), suggesting that R112 mediates a processing-independent role for AvrRps4-N recognition. In accordance with their lack of recognition, 
A Lettuce cv. Kordaat

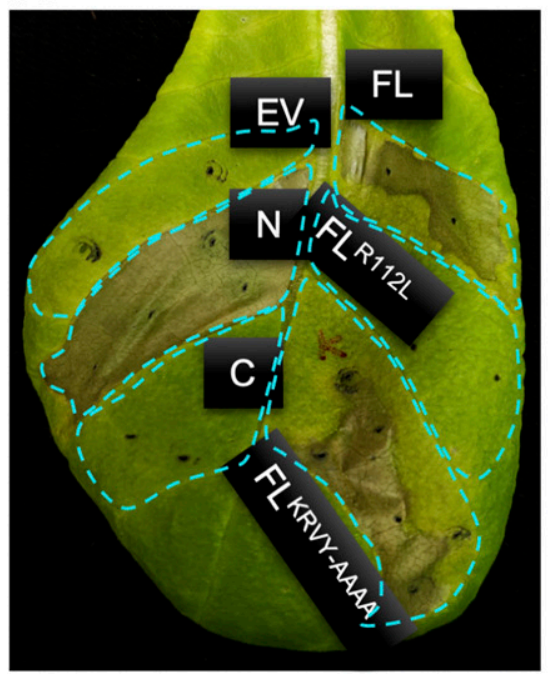

B

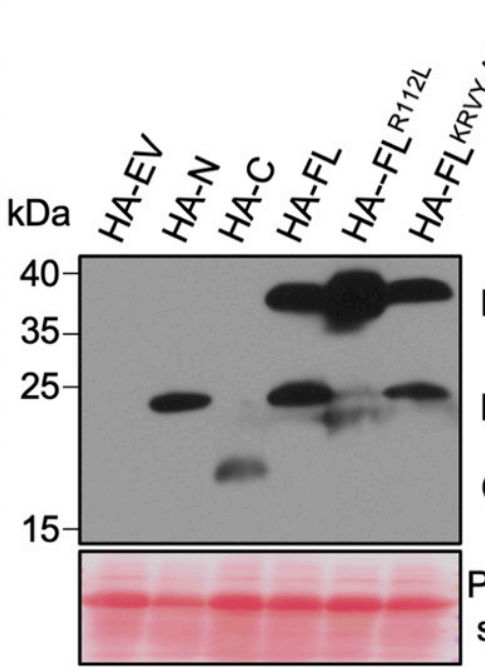

C Turnip (PI 227296)

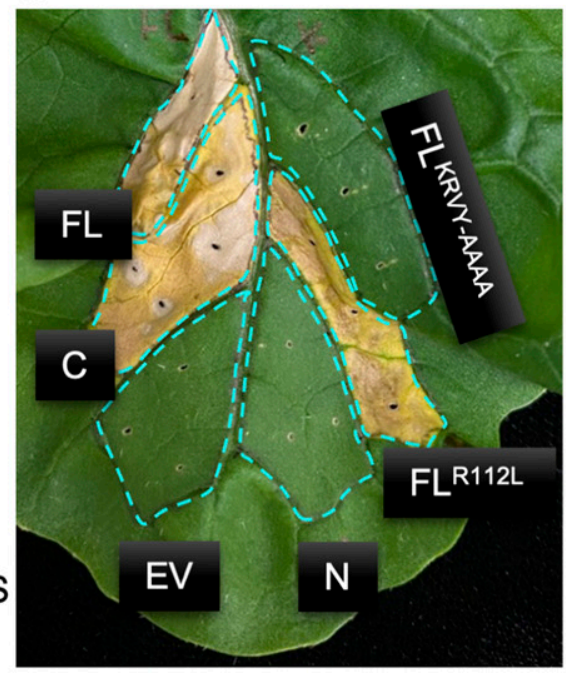

Fig. 1. Differential recognition of AvrRps4 by lettuce and turnip. A, Agrobacterium C58C1 containing the constructs for transient expression of N-terminally hemagglutinin-tagged AvrRps4 full-length (HA-FL), AvrRps4-N (HA-N), AvrRps4-C (HA-C), full-length AvrRps4 ${ }^{\text {112L }}$ (HA-FL ${ }^{\text {R112L }}$ ), full-length AvrRps4 134-137 KRVY to AAAA mutant (HA-FL KRVY-AAAA), and empty vector (HA-EV) was infiltrated into Lactuca sativa cv. Kordaat at an optical density (OD) of 0.4. Photos were taken 3 days postinfiltration (dpi). These experiments were performed at least three times with identical results. B, Protein expression in Kordaat described in A was confirmed by Western blots. Ponceau S staining confirmed equal loading. Samples were harvested at $36 \mathrm{~h}$ postinfiltration, before the occurrence of the hypersensitive response. C, Agrobacterium $\mathrm{C} 58 \mathrm{C} 1$ containing the constructs described in A was infiltrated into turnip Brassica rapa cv. PI227296 at an OD of 0.4. Photos were taken 6 dpi. These experiments were performed twice with identical results.

$\underline{A}$

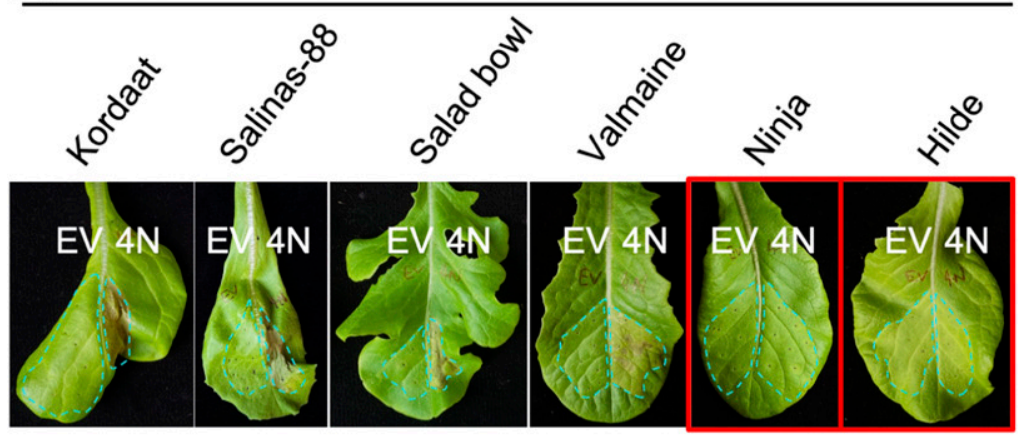

B

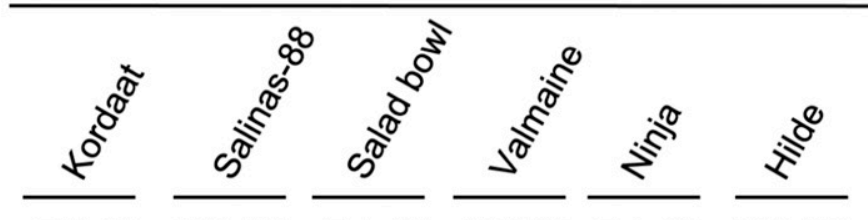

EV 4N EV 4N EV 4N EV 4N EV 4N EV 4N
C

Lactuca serriola

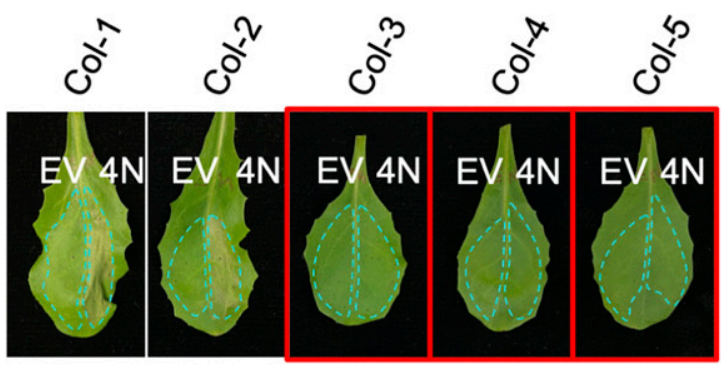

D

Lactuca serriola

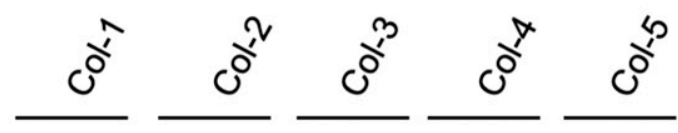

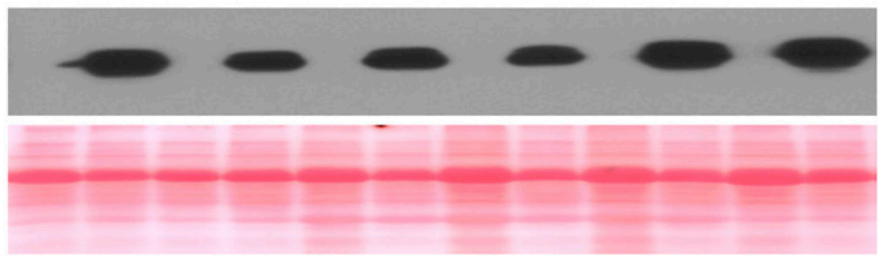

\section{$\mathrm{HA}-4 \mathrm{~N}$ \\ Ponceau S staining}

Fig. 2. Recognition of AvrRps4-N in lettuce cultivars and wild lettuce. A, Agrobacterium C58C1 containing the N-terminally hemagglutinin (HA)-tagged AvrRps4-N (4N) and empty vector pBA-HA (EV) constructs was infiltrated into six lettuce cultivars (Kordaat, Salinas-88, Salad Bowl, Valmaine, Ninja, and Hilde) at an optical density (OD) of 0.4. Photos were taken 3 days postinfiltration (dpi). These experiments were performed twice with identical results. B, Protein expression in Lactuca sativa cultivars was confirmed by Western blots. Ponceau S staining confirmed equal loading. Samples were harvested at $36 \mathrm{~h}$ postinfiltration (hpi) before the occurrence of the hypersensitive response (HR). C, Agrobacterium C58C1 containing the N-terminally HA-tagged AvrRps4-N (4N) and empty vector pBA-HA (EV) constructs was infiltrated into five wild lettuce lines (Lactuca serriola, Col-1, Col-2, Col-3, Col-4, and Col-5) at an OD of 0.4. Photos were taken 3 dpi. These experiments were performed twice with identical results. D, Protein expression in wild lettuce (Lactuca serriola) was confirmed by Western blots. Ponceau S staining confirmed equal loading. Samples were harvested at 36 hpi, before the occurrence of HR. 
AvrRps4-N ${ }^{\mathrm{R} 112 \mathrm{~L}}$ and AvrRps4-FL ${ }^{\mathrm{R} 112 \mathrm{~L}}$ accumulated to a much higher level than AvrRps4-N and AvrRps4-FL, respectively (Fig. 3B). Because arginine is positively charged at physiological $\mathrm{pH}$, we hypothesized that the positive charge of arginine is responsible for AvrRps4-N recognition. To investigate this possibility, we mutated R112 to positively charged lysine and negatively charged glutamate (Fig. 4A). As expected, mutation of R112 to negatively charged glutamate failed to trigger HR. The R112K substitution failed to trigger HR at 2 days postinfiltration (dpi) (Fig. 4B and C). A slow and weak HR by $\mathrm{R} 112 \mathrm{~K}$ is observed only after 6 dpi (Supplementary Fig. S2).

To quantify the level of HR, we performed electrolyte leakage assays. As is shown, wild-type AvrRps4-N induced the highest conductivity, while R112L and R112E substitutions triggered a level of conductivity comparable to that caused by empty vector (Fig. 4D). Consistent with the slow and weak HR, the R112K substitution induced intermediate conductivity (Fig. 4D). Even though both arginine and lysine are positively charged amino acids, they do differ in some biochemical properties; for example, the side chain of arginine is longer and more positively charged than that of lysine, and the guanidinium group in arginine has three possible directions of interaction (Fig. 4A). This allows arginine to form more salt bridges and hydrogen bonds, while the $\varepsilon$-amino group in lysine has only one direction of interaction (Kumar and Nussinov 1999; Matsutani et al. 2011; Musafia et al. 1995; Sokalingam et al. 2012; Strub et al. 2004). These results suggest an R112specific role for AvrRps4-N recognition in lettuce, possibly resulting from its long and positive side chain.

As R112L substitution leads to inefficient AvrRps4 processing, we next examined the effect of R112E and R112K substitutions on AvrRps4 processing. As is shown in Figure 5, AvrRps4-FL ${ }^{\mathrm{R} 12 \mathrm{~L}}$, AvrRps4-FL ${ }^{\mathrm{R} 12 \mathrm{E}}$, and AvrRps4-FL ${ }^{\mathrm{R} 12 \mathrm{~K}}$ all failed to be processed in two lettuce cultivars, Kordaat and Ninja. This result also suggests an R112-specific role for AvrRps 4 processing. Because AvrRps4-N is not recognized in Ninja, the similar processing pattern in Kordaat and Ninja suggests R112-dependent AvrRps4 full-length processing is independent of R112-dependent AvrRps4-N recognition.

\section{The central conserved region of AvrRps4-N is responsible for its recognition in lettuce.}

We next aimed to define which region of AvrRps4-N is responsible for HR induction. In the alignment of orthologs of AvrRps4-N, HopK1-N, and XopO-N, we noticed a conserved C-terminal double glycine motif (Fig. 6A). The C-terminal Gly-Gly motif is considered a key feature of processed ubiquitin and ubiquitin-like proteins (UBLs), such as small ubiquitin-related modifiers (SUMOs), in which it is required for their conjugation to the lysine residue of a target protein (Flotho and Melchior 2013; Komander and Rape 2012). To test whether the Gly-Gly motif is also required for AvrRps4-N recognition, we deleted the last three amino acids of AvrRps4-N to generate AvrRps4-N ${ }^{1-130}$. After agroinfiltration of Kordaat, AvrRps4-N ${ }^{1-130}$ triggered $\mathrm{HR}$ comparable to that of full-length AvrRps4-N, even though AvrRps4-N ${ }^{1-130}$ expressed less than AvrRps4-N (Supplementary Fig. S3), suggesting the C-terminal Gly-Gly motif of AvrRps4-N is not critical for its recognition in lettuce.

We next truncated AvrRps4-N from its C-terminus to generate HA-AvrRps4-N ${ }^{1-126}$, HA-AvrRps4- ${ }^{1-120}$, HAAvrRps4-N ${ }^{1-11}$, HA-AvrRps4-N ${ }^{1-99}$, HA-AvrRps4-N ${ }^{1-93}$, and HA-AvrRps4-N ${ }^{1-79}$. HA-AvrRps4-N ${ }^{1-120}$ still induced HR (Fig. $6 \mathrm{~B}$ and $\mathrm{C}$ ), suggesting that the $\mathrm{C}$-terminal 13 amino acids are not required for AvrRps4-N recognition. HA-AvrRps4- $\mathrm{N}^{1-111}$ HA-AvrRps4-N ${ }^{1-99}$, HA-AvrRps4-N ${ }^{1-93}$, and HA-AvrRps4-N ${ }^{1-79}$ were all expressed well; nevertheless, none of them triggered HR (Fig. 6B and C). We noticed the N-terminal 83 amino acids of AvrRps4-N are less conserved (Fig. 6A). Thus, for $\mathrm{N}$ terminal truncations, we first removed the N-terminal 83 amino acids to produce HA-Flag-HA-AvrRps4-N ${ }^{84-133}$ and, further, removed an additional 20 amino acids to produce HA-Flag-HA-AvrRps4-N ${ }^{104-133}$. As was expected, HA-FlagHA-AvrRps4-N ${ }^{84-133}$ still induced HR while HA-Flag-HAAvrRps4-N ${ }^{104-133}$ failed (Fig. 6D), suggesting that the N-terminal 83 amino acids are dispensable for AvrRps4-N recognition.

Despite repeated use of several protein extraction protocols, we failed to detect protein expression of AvrRps4-N ${ }^{84-133}$ and AvrRps4-N ${ }^{104-133}$ in Kordaat, either by anti-HA or antiFlag antibody, indicating that amino acids 1 to 83 play a role in stabilizing AvrRps4-N (Fig. 6E). Therefore, we tried the DEXinducible expression system and generated a series of truncations: DEX-HA-AvrRps4-N ${ }^{1-46}$, DEX-HA-AvrRps4-N ${ }^{4-133}$, DEX-HA-AvrRps4-N ${ }^{1-83}$, DEX-HA-AvrRps4-N ${ }^{84-133}$, DEXHA-AvrRps4-N ${ }^{47-83}$, and DEX-HA-AvrRps4-N ${ }^{1-133}$ 'Supplementary Fig. S4A). Consistent with the previous deletion series, DEX-HA-AvrRps4-N ${ }^{84-133}$ triggered HR while DEX-HAAvrRps4-N ${ }^{1-83}$ did not (Supplementary Fig. S4B). Protein expression of DEX-HA-AvrRps4-N ${ }^{1-46}$, DEX-HA-AvrRps4-N ${ }^{47-83}$, DEX-HA-AvrRps4-N ${ }^{47-133}$, and DEX-HA-AvrRps4-N ${ }^{84-133}$ was at or below the detection limit in lettuce. Transient expression in Nicotiana benthamiana, in which no HR interferes with protein accumulation, protein expression could be detected for all constructs, albeit at very low levels for DEX-HA-AvrRps4-N ${ }^{84-133}$

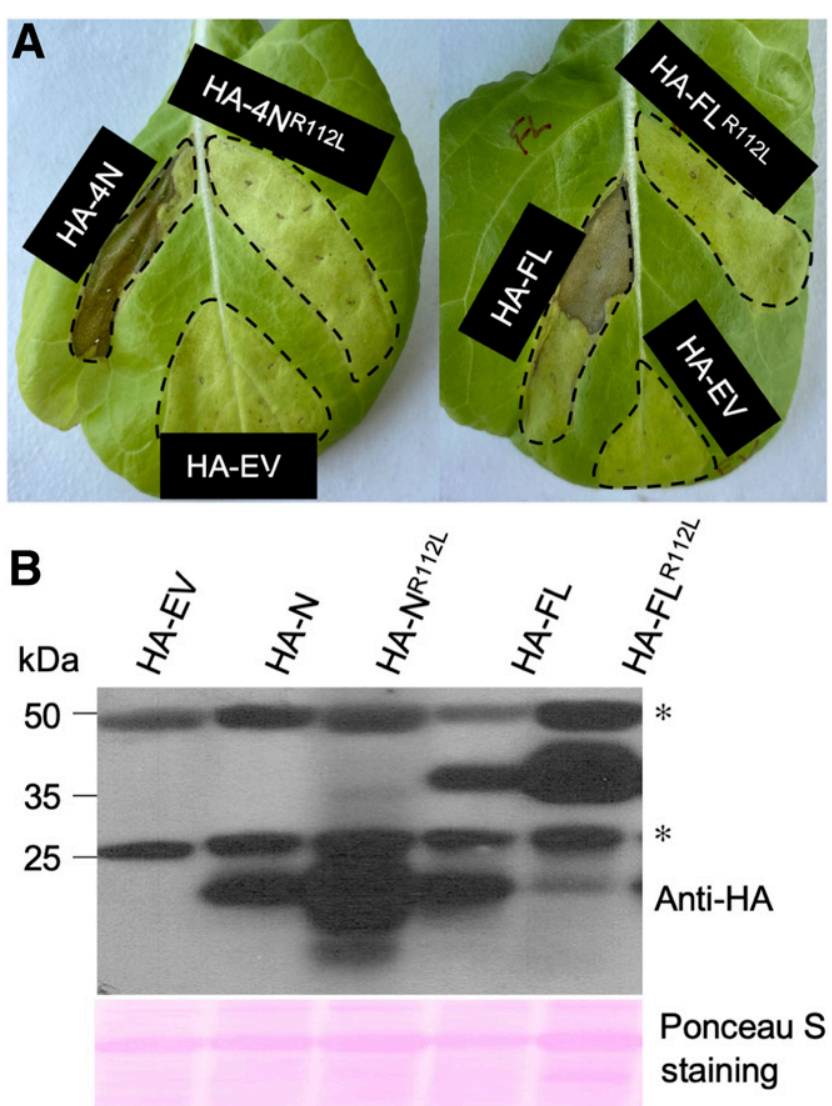

Fig. 3. R112 is required for AvrRps4-FL processing and, also, for AvrRps4$\mathrm{N}$ recognition. A, N-terminally hemagglutinin (HA)-tagged full-length AvrRps4 (HA-FL), full-length AvrRps4 ${ }^{\mathrm{R} 112 \mathrm{~L}}$ (HA-FL ${ }^{\mathrm{R} 112 \mathrm{~L}}$ ), AvrRps4-N (HA-N), AvrRps4-N ${ }^{R 112 L}$ (HA-N ${ }^{R 12 L}$ ), and empty-vector HA-pBA (HAEV) were expressed in Kordaat at an optical density of 0.4. Cell death was imaged 3 days postinfiltration. This experiment was performed three times with identical results. B, Protein expression in Kordaat was confirmed by Western blots. Ponceau S staining confirmed equal loading. Asterisks indicate nonspecific antibody binding. 
and DEX-HA-AvrRps4-N ${ }^{4-83}$ (Supplementary Fig. S4C), further suggesting that amino acids 1 to 83 function in stabilizing AvrRps4-N. Taken together, the short conserved central region consisting of 37 amino acids from residues 84 to 120 of AvrRps4-N is necessary and sufficient for its recognition in lettuce.

\section{DISCUSSION}

Fundamental work on the recognition of AvrRps4 by the RRS1/RPS4 and RRS1B/RPS4B TNL protein pairs have focused on the function of AvrRps4-C (Sarris et al. 2015; Saucet et al. 2015). This focus led to the discovery that AvrRps4-C disrupts the intramolecular interaction of the RRS1 WRKY domain with its adjacent domains as a necessary step toward activation of RPS4 and RPS4B (Ma et al. 2018). However, our recent study showing that AvrRps4 is a bipartite effector and that AvrRps4-N but not AvrRps4-C can be recognized by lettuce (Halane et al. 2018) increased the complexity of recognition mechanisms for AvrRps4. To gain further insight into the recognition of AvrRps4-N in this study, we performed serial truncations and point mutagenesis and identified a conserved central region of AvrRps4-N as the determinant for its recognition in lettuce. In addition, AvrRps4-N with a mutation of the conserved amino acid R112 in this region failed to cause HR.
Based on the observed HR-inducing pattern, we concluded that lettuce only recognizes AvrRps4-N while turnip recognizes AvrRps4-C (Fig. 1A and C) (Sohn et al. 2009). However, we found AvrRps4-N and AvrRps4-C are mutually required to trigger ETI in Arabidopsis when delivered by DC3000 (Halane et al. 2018), which conflicts with the finding that estradiolinducible expression of AvrRps4-C alone induces strong HR in Arabidopsis ( $\mathrm{Li}$ et al. 2014). We reason that these inconsistent HR reports may result from the differential protein levels of AvrRps4 or AvrRps4-C in different systems. Thus, it will be interesting to test, in the future, whether AvrRps4-N and AvrRps4-C induce the same responses in lettuce and turnip when delivered from bacteria by the type III secretion system.

In this study, we showed that R112 is required for both AvrRps4-FL processing and AvrRps4-N recognition in lettuce (Fig. 3A and B). The dual role of R112 raises the question whether AvrRps4-FL processing is required for recognition? Here, we propose two possible models for AvrRps4-FL processing and AvrRps4-N recognition. i) AvrRps4-FL is processed by one or more peptidases first; then an unknown protein, possibly an R protein, mediates AvrRps4-N recognition (Fig. 7A). In this model, the possible $\mathrm{R}$ protein only recognizes AvrRps4-N but not AvrRps4-FL. ii) AvrRps4-FL interacts with both the peptidase or peptidases and the possible R protein in an R112dependent manner (Fig. 7B). In this model, both recognition of

A<smiles>N=C(N)NCCCC(N)C(=O)O</smiles>

Leu<smiles>CC(C)CC(N)C(=O)O</smiles><smiles>NCCCC[C@H](N)C(=O)O</smiles>
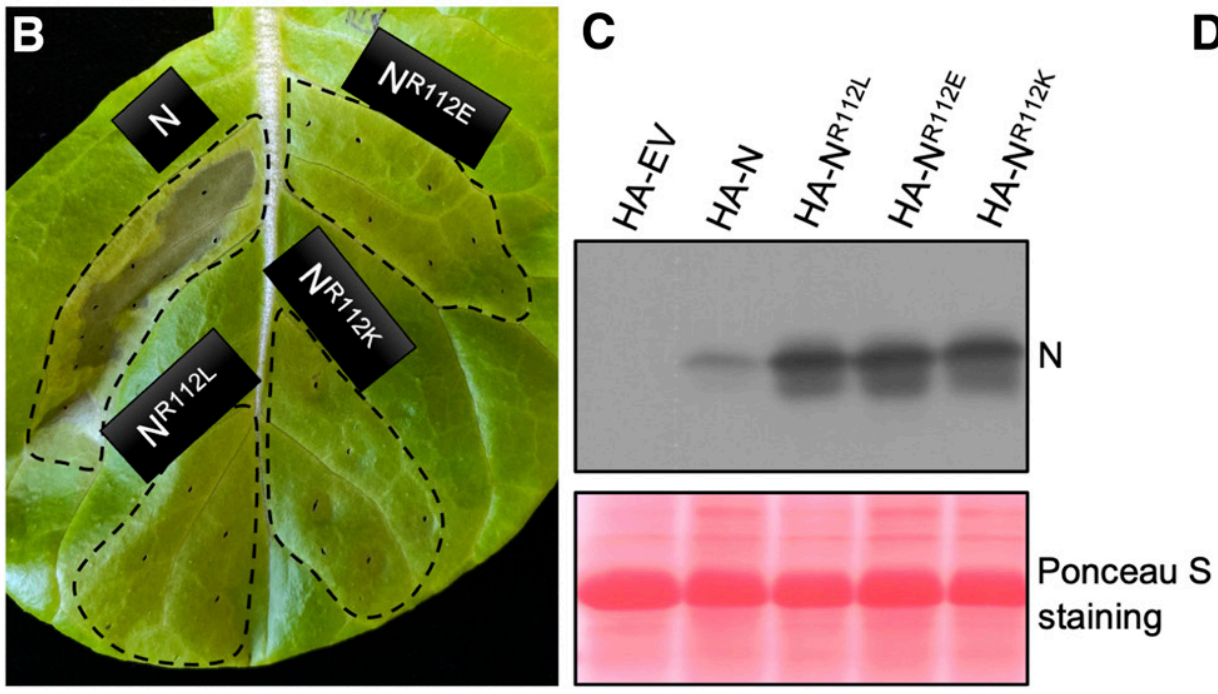<smiles>NC(CCC(=O)O)C(=O)O</smiles>

D

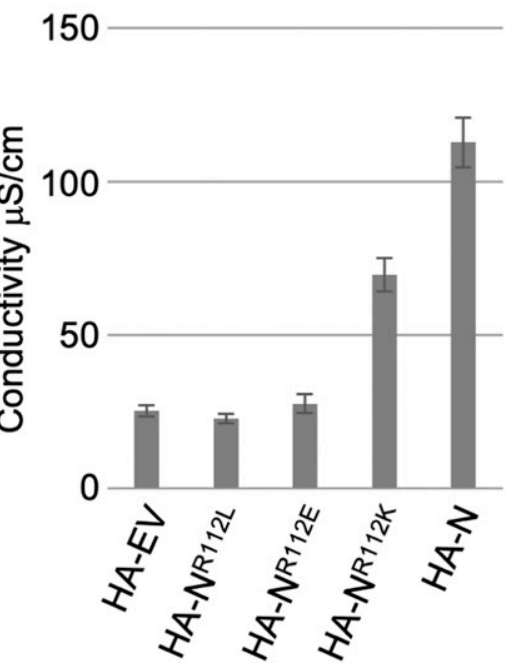

Fig. 4. The positive charge and specific structure of R112 is also required for AvrRps4-N recognition. A, Structural formulas and properties of arginine (Arg), leucine (Leu), lysine (Lys), and glutamic acid (Glu). B, N-terminally hemagglutinin (HA)-tagged AvrRps4-N (HA-N), AvrRps4-N ${ }^{\mathrm{R} 112 \mathrm{~L}}$ (HA-N ${ }^{\mathrm{R} 112 \mathrm{~L}}$ ),

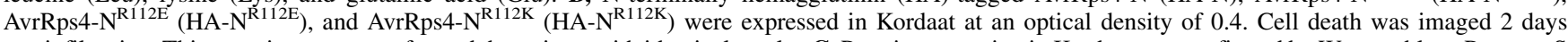
postinfiltration. This experiment was performed three times with identical results. C, Protein expression in Kordaat was confirmed by Western blots. Ponceau $\mathrm{S}$ staining confirmed equal loading. D, Electrolyte release by cells undergoing hypersensitive response was measured at $36 \mathrm{~h}$ postinfiltration in electrolyte leakage assays. Conductivity values are means \pm standard deviation, $n=4$. This experiment was performed twice with similar results. 
AvrRps4-FL and AvrRps4-N by the putative R protein trigger ETI. To test these possibilities, we need to identify one or more peptidases that cleave AvrRps4-FL and the R protein that recognizes AvrRps4-N in lettuce. Our finding that Ninja, Hilde, and three wild lettuce lines fail to recognize AvrRps4-N (Fig. 2A and B) makes it possible to map the gene for AvrRps4-N. The gene for recognizing AvrRps4-FL in Salinas was mapped to a 7-Mb region of chromosome 8 that contains 127 genes, 36 of which are $R$ genes (Christopoulou et al. 2015). Due to the similar HR pattern and the absence of any contribution to HR by AvrRps4-C, it is highly likely that the gene for recognizing AvrRps4-N is identical to the one recognizing AvrRps4-FL and is also localized in this 7-Mb region. In the future, CRISPR-mediated knockout and genetic complementation will be carried out to define the determinant for AvrRps4-N recognition. More broadly, the existence of resistance polymorphisms to AvrRps4-N in wild lettuce supports the proposition that AvrRsp4-N and its homologs represent a bona fide effector function that lettuce coevolved with.

It is interesting that R112 of AvrRps4-N cannot be substituted by the positively charged lysine (Fig. 4B to D), since only a slow and weak HR is observed with R112K (Supplementary Fig. S2). Despite their conserved nature, arginine and lysine are not interchangeable in certain conditions. A well-known example is that ubiquitin and UBLs can only be conjugated to lysine residues but not arginine (Flotho and Melchior 2013; Komander and Rape 2012). Conversely, arginine cannot be replaced by lysine in some cases. For example, mutation of the conserved R42 of a chitosanase from Streptomyces sp. strain N174 into lysine resulted in drastic loss of enzymatic activity (Lacombe-Harvey et al. 2013). Also, an arginine to lysine substitution in a maize bZIP transcriptional factor failed to bind its specific target promoter (Aukerman et al. 1991). Similarly, the R280 to lysine mutation abolishes the DNA binding ability of human p53 protein, a wellknown tumor suppressor (Malcikova et al. 2010). According to a structure study (Gomes et al. 2018), the interaction between R280 and the guanine nucleotide is stronger than that of lysine, as it forms two hydrogen bonds while lysine can only form one hydrogen bond. In addition, the side chain of arginine is longer and is more positively charged than lysine, which favors the interaction of p53 with the DNA molecule (Gomes et al. 2018). Thus, it is reasonable to hypothesize that the long and positively charged side chain of R112 may enable AvrRps4 to interact strongly with the peptidase or peptidases and the putative $\mathrm{R}$ protein that are responsible for its cleavage and recognition (Fig. 7A and B).

The finding that an electronegative surface patch in AvrRps4C, mainly formed by amino acids E175 and E187, was required for RPS4-RRS1- and RPS4B-RRS1B-mediated recognition (Saucet et al. 2015; Sohn et al. 2012) has been supported by subsequent studies that reveal possible mechanisms. First, an AvrRps4 mutant with both E175 and E187 mutated to alanine fails to target RRS1 (Ma et al. 2018). Secondly, interaction of AvrRps4 with RRS1 is compromised when its lysine in the WRKY motif is acetylated; the acetyl groups add negative charges to the positively charged lysine residues (Sarris et al. 2015). Similarly, the essential role of R112 demonstrated by the present study may facilitate revelation of the mechanism of AvrRps4-N-triggerd $\mathrm{R}$ protein activation and of its virulence function. For future studies of AvrRps 4 variants in lettuce, a well-defined lettuce-Pseudomonas pathosystem through which natural levels of AvrRps4-N, AvrRps4-C, and AvrRps4-FL are delivered by the type III secretion system would be beneficial.

\section{MATERIALS AND METHODS}

Plant materials and growth conditions.

Lettuce was grown in SunGro Professional Growing Mix at $22^{\circ} \mathrm{C}$ and $75 \%$ humidity in a walk-in Conviron growth chamber with a 16-h light and 8-h dark cycle and with light intensity around $120 \mu \mathrm{mol} \mathrm{m} \mathrm{m}^{-2} \mathrm{~s}^{-1}$. Lettuce cultivars Kordaat, Ninja, Salad Bowl, Salinas-88, and Hilde were provided by the United States Department of Agriculture National Plant Germplasm System. Seeds of wild lettuce were collected from areas near Columbia, MO, U.S.A. Turnip seeds for PI 227296 and PI 212593 were provided by H. An and J. C. Pires, Division of Biological Sciences, University of Missouri.

\section{Plasmid construction.}

Coding sequences for AvrRps4-N, AvrRps4-C, and AvrRps4FL with or without stop codons were cloned into pDONOR201 via Gateway recombination as previously described (Bhattacharjee et al. 2011). Point mutations of AvrRps4-N $\mathrm{N}^{\mathrm{R} 112 \mathrm{~L}}$, AvrRps4-FL ${ }^{\text {Ri 12L }}$, and AvrRps4-FL KRVY-AAAA were introduced via site-directed mutagenesis (Liu and Naismith 2008; Sohn et al. 2009). N-terminally HA-tagged constructs were generated as previously described (Bhattacharjee et al. 2011). To make AvrRps4-N C-terminal truncations, stop codons were introduced into the pBA-HA-AvrRps4-N construct at 80, 94, 100, 112,121 , and 127 to produce AvrRps4- $\mathrm{N}^{1-79}$, AvrRps4-N ${ }^{1-93}$, AvrRps4-N ${ }^{1-99}$ AvrRps4-N ${ }^{1-11}$, AvrRps4-N ${ }^{1-120}$, and AvrRps4- ${ }^{1-126}$. To generate N-terminal AvrRps4-N truncations, coding sequences for BamHI-HA-Flag-HA-AvrRps4$\mathrm{N}^{84-133}-$ SacI and BamHI-HA-Flag-HA-AvrRps4-N ${ }^{104-133}-$ SacI were directly synthesized into pIDT-Kan. Then the fragments were digested with $\mathrm{BamHI}$ and $\mathrm{SacI}$ and were subcloned into pBA-HA vector. To generate the DEX-inducible AvrRps4-N constructs, a modified multisite Gateway system (Invitrogen) was used, as described previously (Kim et al. 2016). Briefly, AvrRps4-N and its deletion derivatives ( 1 to 46,47 to 133,1 to 83,84 to 133,47 to 83 ) were PCR-amplified from a wild-type AvrRps4 plasmid template and were cloned into pDONORII

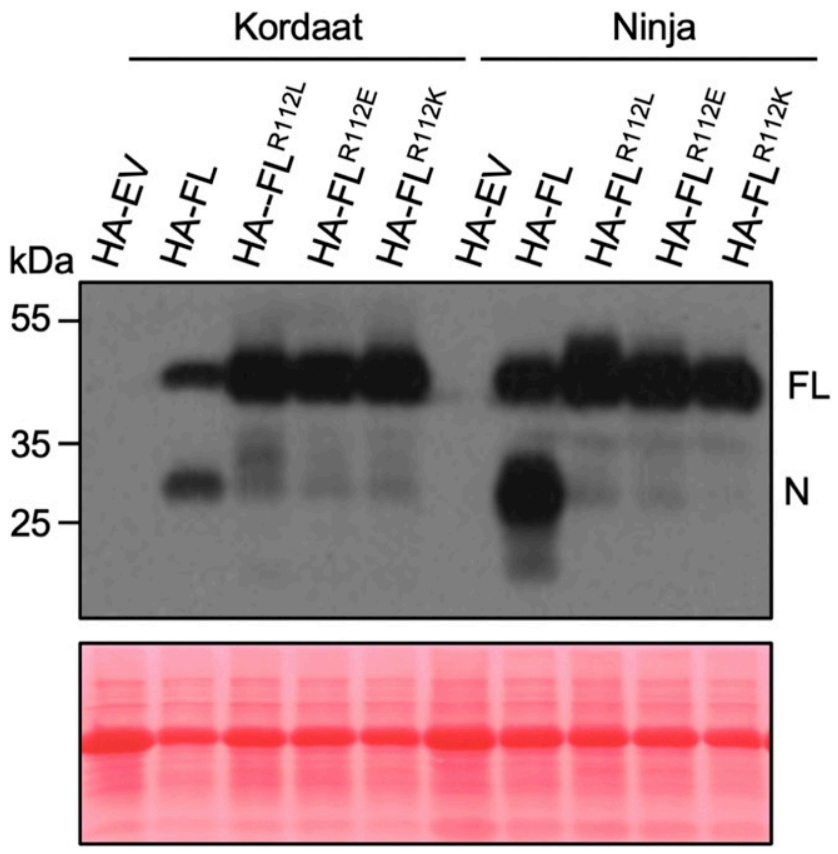

Fig. 5. R112-dependent AvrRps4 cleavage is independent of its recognition. N-terminally hemagglutinin (HA)-tagged full-length AvrRps4 (HA-FL), AvrRps4-FL ${ }^{\mathrm{R} 112 \mathrm{~L}}$ (HA-FL ${ }^{\mathrm{R} 112 \mathrm{~L}}$ ), AvrRps4-FL ${ }^{\mathrm{R} 112 \mathrm{E}}$ (HA-FL ${ }^{\mathrm{R} 12 \mathrm{E}}$ ), AvrRps4-FL ${ }^{\mathrm{R} 112 \mathrm{~K}}$ (HA-FL ${ }^{\mathrm{R} 112 \mathrm{~K}}$ ), and empty-vector HA-pBA (HA-EV) were expressed in Kordaat and Ninja at an optical density of 0.4. Samples were collected at $36 \mathrm{~h}$ postinfiltration. AvrRps 4 cleavage was detected by Western blots. Ponceau S staining confirmed equal loading (lower panel). 
using BP reactions. These entry clones and pDONRI:3 $\times \mathrm{HA}$ were recombined into the pTA7002 destination vector (carrying a DEX-inducible promoter), using LR reactions to generate the $\mathrm{N}$-terminally HA-tagged constructs. All constructs were confirmed by sequencing. Primer sequences used in cloning are listed in Supplementary Table S1.
Agrobacterium-mediated infiltration.

All plasmids in this study were transformed into Agrobacterium tumefaciens $\mathrm{C} 58 \mathrm{C} 1$, using electroporation. For HR assays in lettuce and turnip, C58C1 carrying corresponding constructs and empty vector were recovered from stock by streaking on Luria-Bertani (Miller's formulation) (LB) plates
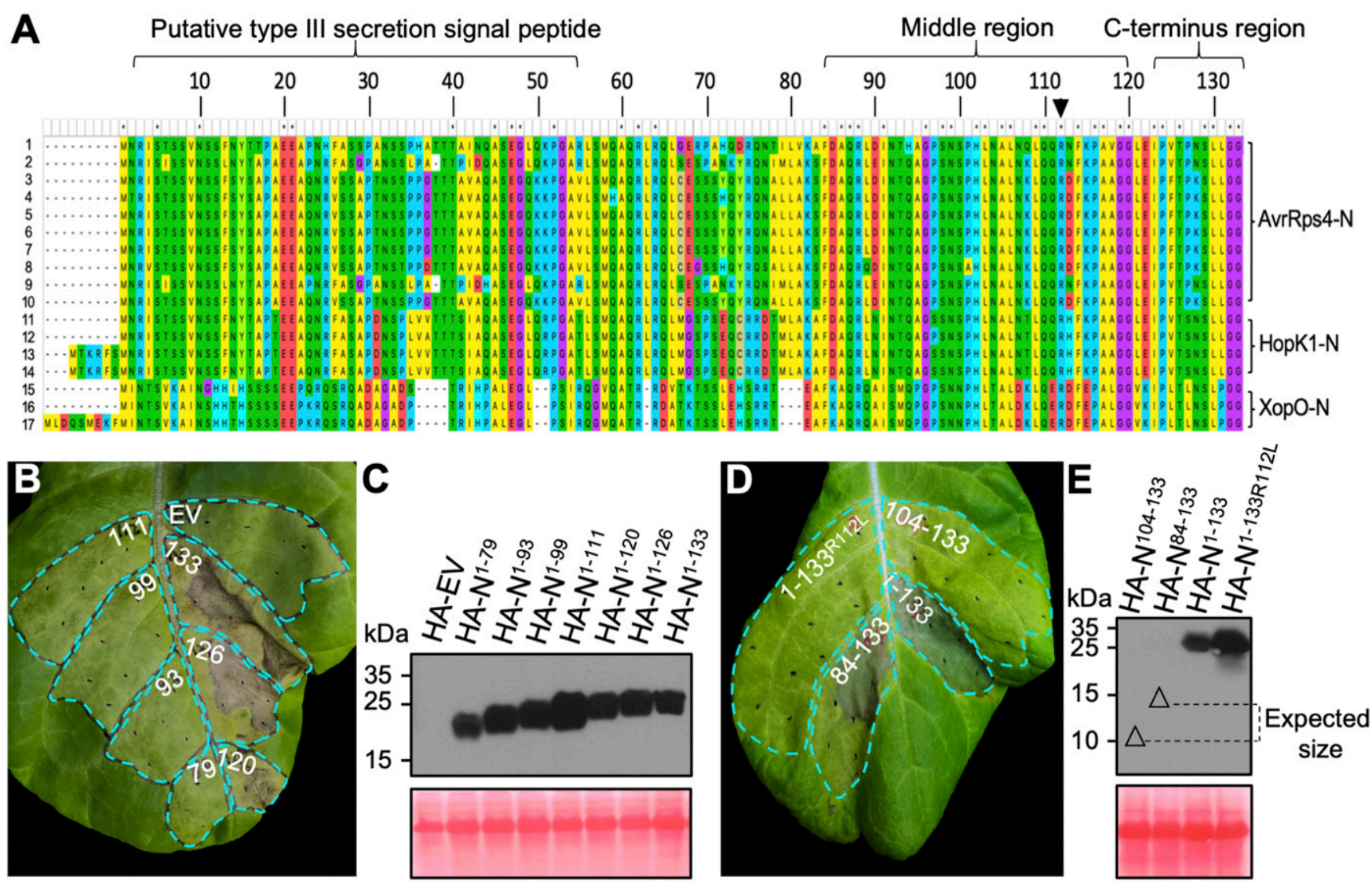

Fig. 6. The central conserved region of AvrRps4-N is required for its recognition. A, Alignment of AvrRps4-N, HopK1-N, and XopO-N orthologs by MEGA-X software. Sequences for AvrRps4-N: 1 = KGK92129.1, 2 = KOP53052.1, 3 = KPX03623.1, 4 = PYD08707.1, $5=$ RMQ54834.1, $6=$ RMQ56798.1, $7=$ RMR22859.1, $8=$ WP 116834360.1, $9=$ RMS14090.1, and 10 = RMU85203.1; for HopK1-N: $11=$ KPB91745.1, 12= RMR21473.1, 13= KPW70528.1, and $14=$ KPY92535.1; and for XopO-N: $15=$ AEQ95299.1, $16=$ WP_127172224.1, and 17 = AAV74207.1. Alignment was performed using MEGA 10.1.7, MUSULE. An asterisk (*) indicates identical amino acids, the black arrow highlights the conserved R112 in AvrRps4-N. B, N-terminally hemagglutinin (HA)tagged AvrRps4-N (133), AvrRps4-N1-126 (126), AvrRps4-N1-120 (120), AvrRps4-N1-111 (111), AvrRps4-N1-99 (99), AvrRps4-N1-93 (93), AvrRps4-N179 (79), and empty vector pBA-HA (EV) were expressed in Kordaat at an optical density (OD) of 0.4. The photo was taken 3 days postinfiltration (dpi). This experiment was performed at least three times with identical results. C, Protein expression of AvrRps4-N truncations in Kordaat was confirmed by Western blots. Ponceau S staining confirmed equal loading (lower panel). D, N-terminally HA-tagged AvrRps4-N ${ }^{104-133}$ (104-133), AvrRps4-N ${ }^{84-133}$ (84-133), AvrRps4-N $\mathrm{N}^{1-133}(1-133)$, and AvrRps4-N $\mathrm{N}^{1-133 \mathrm{R} 112 \mathrm{~L}}(1-133 \mathrm{R} 112 \mathrm{~L})$ were expressed in Kordaat at an OD of 0.4. The photo was taken 3 dpi. This experiment was performed at least three times with identical results. E, Protein expression of AvrRps4-N truncations in Kordaat was confirmed by Western blots. Triangles ( $\triangle$ ) indicate the expected bands. Ponceau S staining confirmed equal loading (lower panel).

A

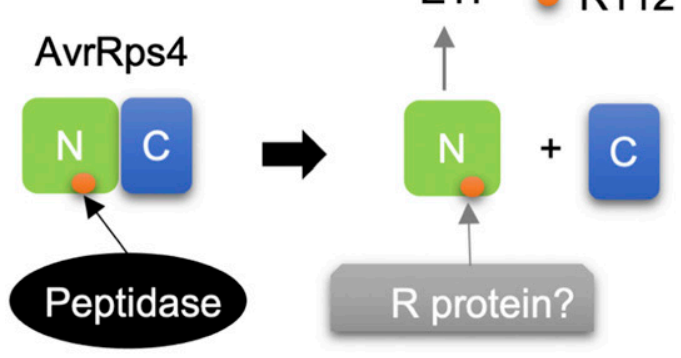

B

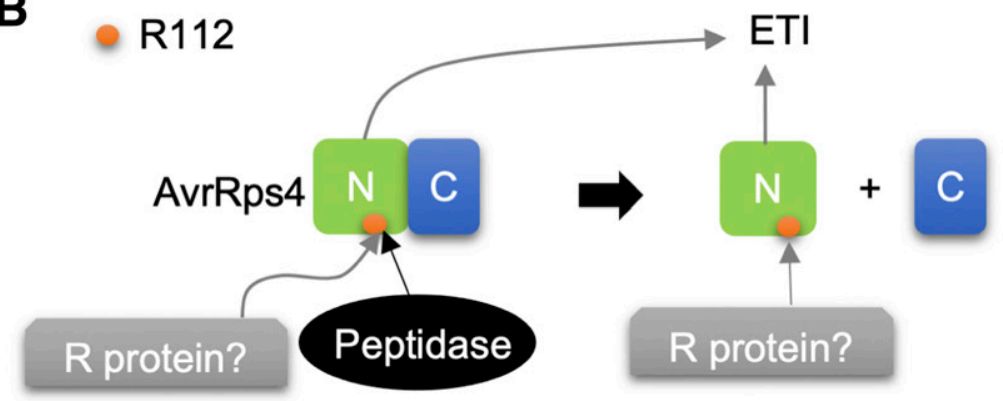

Fig. 7. Two possible models for R112-dependent AvrRps4-N recognition in lettuce. A, AvrRps4-FL processing is required for AvrRps4-N recognition. Both are dependent on R112. B, R112-mediated AvrRps4-FL processing and AvrRps4-N recognition are independent. In this model, the putative resistance (R) protein can recognize both AvrRps4-FL and AvrRps4-N. 
with appropriate antibiotics. For each construct, about five colonies were transferred to liquid medium and were grown at $30^{\circ} \mathrm{C}$ with $180 \mathrm{rpm}$ shaking. After overnight culture, cells were collected via centrifugation at $3,000 \times g$ at room temperature. Pellets were washed twice to remove residual LB media, then, were resuspended in a buffer with $10 \mathrm{mM}$ MES (pH 5.6), $10 \mathrm{mM} \mathrm{MgCl}$, and $100 \mu \mathrm{M}$ acetosyringone. Suspensions were kept at room temperature for $4 \mathrm{~h}$ before adjusting the optical density to 0.4 . HR phenotypes were visualized 2 to 3 dpi unless otherwise noted. For DEX-inducible expression 2 dpi, infiltrated leaves were sprayed with a 50- $\mu \mathrm{M}$ DEX solution. Photos were taken under UV light and white light 1 day after DEX treatment. For Western blot, tissues were collected $4 \mathrm{~h}$ after DEX treatment.

\section{Electrolyte leakage assay.}

For electrolyte leakage assays, infiltrated leaves were infiltrated with sterile deionized water again at $36 \mathrm{~h}$ postinfiltration (hpi); then, 10 leaf discs were randomly excised and were transferred into a vial containing $10 \mathrm{ml}$ of deionized water. Conductivity was measured $1 \mathrm{~h}$ later with a PC850 portable conductivity meter (Apera). Four replicates were measured for each construct.

\section{Protein sequence alignment.}

Protein sequences for AvrRps4-N, HopK1-N, and XopO-N were aligned using MEGA 10.1.7, MUSULE program (Stecher et al. 2020). AvrRps4-N, HopK1-N, and XopO-N were extracted from full-length proteins as follows: AvrRps4-N: i) KGK92129. 1, ii) KOP53052.1, iii) KPX03623.1, iv) PYD08707.1, v) RMQ54834.1, vi) RMQ56798.1, vii) RMR22859.1, viii) WP 116834360.1, ix) RMS14090.1, and x) RMU85203.1; HopK1-N: xi) KPB91745.1, xii) RMR21473.1, xiii) KPW70528.1, and xiv) KPY92535.1; XopO-N: xv) AEQ95299.1, xvi) WP_127172224.1 and xvii) AAV74207.1.

\section{Protein extraction and Western blot.}

Lettuce leaf discs ( $1 \mathrm{~cm}$ in diameter) were collected at $36 \mathrm{hpi}$, before the development of clear HR. Generally, 10 leaf discs collected from three independent plants were combined and total protein was extracted with $250 \mu$ lof protein extraction buffer containing $100 \mathrm{mM}$ Tris- $\mathrm{HCl}$ (pH6.8), 4\% (wt/vol) sodium dodecyl sulfate, $20 \%$ (vol/vol) glycerol, and $100 \mathrm{mM}$ dithiothreitol. After centrifugation at $12,000 \times g$ at $4^{\circ} \mathrm{C}, 200 \mu \mathrm{l}$ of supernatant was transferred into a new tube with $50 \mu$ of $5 x$ loading dye. Protein samples were boiled for $10 \mathrm{~min}$ before loading. For detection, immunoblotting was performed using $\alpha$ HA-HRP (clone 3F10) (Roche) with 1:3,000 dilution.

\section{ACKNOWLEDGMENTS}

We thank R. Michelmore, University of California, Davis, for initial provisioning of lettuce seeds, and H. An and J. C. Pires, University of Missouri, for providing turnip seeds.

\section{LITERATURE CITED}

Aukerman, M. J., Schmidt, R. J., Burr, B., and Burr, F. A. 1991. An arginine to lysine substitution in the bZIP domain of an opaque-2 mutant in maize abolishes specific DNA binding. Genes Dev. 5:310-320.

Bhattacharjee, S., Halane, M. K., Kim, S. H., and Gassmann, W. 2011. Pathogen effectors target Arabidopsis EDS1 and alter its interactions with immune regulators. Science 334:1405-1408.

Block, A., and Alfano, J. R. 2011. Plant targets for Pseudomonas syringae type III effectors: Virulence targets or guarded decoys? Curr. Opin. Microbiol. 14:39-46.

Christopoulou, M., Wo, S. R., Kozik, A., McHale, L. K., Truco, M. J., Wroblewski, T., and Michelmore, R. W. 2015. Genome-wide architecture of disease resistance genes in lettuce. G3 (Bethesda) 5:2655-2669.
Cui, H., Tsuda, K., and Parker, J. E. 2015. Effector-triggered immunity: From pathogen perception to robust defense. Annu. Rev. Plant Biol. 66: 487-511.

Feng, F., and Zhou, J.-M. 2012. Plant-bacterial pathogen interactions mediated by type III effectors. Curr. Opin. Plant Biol. 15:469-476.

Flotho, A., and Melchior, F. 2013. Sumoylation: A regulatory protein modification in health and disease. Annu. Rev. Biochem. 82:357-385.

Gassmann, W. 2005. Natural variation in the Arabidopsis response to the avirulence gene hopPsyA uncouples the hypersensitive response from disease resistance. Mol. Plant-Microbe Interact. 18:1054-1060.

Gassmann, W., Hinsch, M. E., and Staskawicz, B. J. 1999. The Arabidopsis RPS4 bacterial-resistance gene is a member of the TIR-NBS-LRR family of disease-resistance genes. Plant J. 20:265-277.

Gomes, A. S., Trovão, F., Andrade Pinheiro, B., Freire, F., Gomes, S., Oliveira, C., Domingues, L., Romão, M. J., Saraiva, L., and Carvalho, A. L. 2018. The crystal structure of the R280K mutant of human p53 explains the loss of DNA binding. Int. J. Mol. Sci. 19:1184-1195.

Halane, M. K., Kim, S. H., Spears, B. J., Garner, C. M., Rogan, C. J., Okafor, E. C., Su, J., Bhattacharjee, S., and Gassmann, W. 2018. The bacterial type III-secreted protein AvrRps4 is a bipartite effector. PLoS Pathog. 14:e1006984.

Heidrich, K., Wirthmueller, L., Tasset, C., Pouzet, C., Deslandes, L., and Parker, J. E. 2011. Arabidopsis EDS1 connects pathogen effector recognition to cell compartment-specific immune responses. Science 334:1401-1404.

Hinsch, M., and Staskawicz, B. 1996. Identification of a new Arabidopsis disease resistance locus, RPS4, and cloning of the corresponding avirulence gene, avrRps4, from Pseudomonas syringae pv. pisi. Mol. Plant-Microbe Interact. 9:55-61.

Jones, J. D. G., and Dangl, J. L. 2006. The plant immune system. Nature 444:323-329.

Kim, S. H., Qi, D., Ashfield, T., Helm, M., and Innes, R. W. 2016. Using decoys to expand the recognition specificity of a plant disease resistance protein. Science 351:684-687.

Komander, D., and Rape, M. 2012. The ubiquitin code. Annu. Rev. Biochem. 81:203-229.

Kourelis, J., and van der Hoorn, R. A. L. 2018. Defended to the nines: 25 years of resistance gene cloning identifies nine mechanisms for $\mathrm{R}$ protein function. Plant Cell 30:285-299.

Kumar, S., and Nussinov, R. 1999. Salt bridge stability in monomeric proteins. J. Mol. Biol. 293:1241-1255.

Lacombe-Harvey, M.-È., Fortin, M., Ohnuma, T., Fukamizo, T., Letzel, T., and Brzezinski, R. 2013. A highly conserved arginine residue of the chitosanase from Streptomyces sp. N174 is involved both in catalysis and substrate binding. BMC Biochem. 14:23.

Li, G., Froehlich, J. E., Elowsky, C., Msanne, J., Ostosh, A. C., Zhang, C., Awada, T., and Alfano, J. R. 2014. Distinct Pseudomonas type-III effectors use a cleavable transit peptide to target chloroplasts. Plant J. 77: 310-321.

Liu, H., and Naismith, J. H. 2008. An efficient one-step site-directed deletion, insertion, single and multiple-site plasmid mutagenesis protocol. BMC Biotechnol. 8:91.

Lolle, S., Stevens, D., and Coaker, G. 2020. Plant NLR-triggered immunity: From receptor activation to downstream signaling. Curr. Opin. Immunol. 62:99-105.

Ma, Y., Guo, H., Hu, L., Martinez, P. P., Moschou, P. N., Cevik, V., Ding, P., Duxbury, Z., Sarris, P. F., and Jones, J. D. G. 2018. Distinct modes of derepression of an Arabidopsis immune receptor complex by two different bacterial effectors. Proc. Natl. Acad. Sci. U.S.A. 115: 10218-10227.

Malcikova, J., Tichy, B., Damborsky, J., Kabathova, J., Trbusek, M., Mayer, J., and Pospisilova, S. 2010. Analysis of the DNA-binding activity of p53 mutants using functional protein microarrays and its relationship to transcriptional activation. Biol. Chem. 391:197-205.

Matsutani, M., Hirakawa, H., Nishikura, M., Soemphol, W., Ali, I. A. I., Yakushi, T., and Matsushita, K. 2011. Increased number of argininebased salt bridges contributes to the thermotolerance of thermotolerant acetic acid bacteria, Acetobacter tropicalis SKU1100. Biochem. Biophys. Res. Commun. 409:120-124.

Musafia, B., Buchner, V., and Arad, D. 1995. Complex salt bridges in proteins: Statistical analysis of structure and function. J. Mol. Biol. 254: 761-770.

Narusaka, M., Shirasu, K., Noutoshi, Y., Kubo, Y., Shiraishi, T., Iwabuchi, M., and Narusaka, Y. 2009. RRS1 and RPS4 provide a dual Resistancegene system against fungal and bacterial pathogens. Plant J. 60:218-226.

Reyes-Chin-Wo, S., Wang, Z., Yang, X., Kozik, A., Arikit, S., Song, C., Xia, L., Froenicke, L., Lavelle, D. O., Truco, M.-J., Xia, R., Zhu, S., Xu, C., Xu, H., Xu, X., Cox, K., Korf, I., Meyers, B. C., and Michelmore, 
R. W. 2017. Genome assembly with in vitro proximity ligation data and whole-genome triplication in lettuce. Nat. Commun. 8:14953.

Sarris, P. F., Duxbury, Z., Huh, S. U., Ma, Y., Segonzac, C., Sklenar, J., Derbyshire, P., Cevik, V., Rallapalli, G., Saucet, S. B., Wirthmueller, L., Menke, F. L. H., Sohn, K. H., and Jones, J. D. G. 2015. A plant immune receptor detects pathogen effectors that target WRKY transcription factors. Cell 161:1089-1100.

Saucet, S. B., Ma, Y., Sarris, P. F., Furzer, O. J., Sohn, K. H., and Jones, J. D. 2015. Two linked pairs of Arabidopsis TNL resistance genes independently confer recognition of bacterial effector AvrRps4. Nat. Commun. 6:6338.

Sohn, K. H., Hughes, R. K., Piquerez, S. J., Jones, J. D. G., and Banfield, M. J. 2012. Distinct regions of the Pseudomonas syringae coiled-coil effector AvrRps4 are required for activation of immunity. Proc. Natl. Acad. Sci. U.S.A. 109:16371-16376.

Sohn, K. H., Zhang, Y., and Jones, J. D. G. 2009. The Pseudomonas syringae effector protein, AvrRPS4, requires in planta processing and the KRVY domain to function. Plant J. 57:1079-1091.

Sokalingam, S., Raghunathan, G., Soundrarajan, N., and Lee, S.-G. 2012. A study on the effect of surface lysine to arginine mutagenesis on protein stability and structure using green fluorescent protein. PLoS One 7:e40410.

Spoel, S. H., and Dong, X. 2012. How do plants achieve immunity? Defence without specialized immune cells. Nat. Rev. Immunol. 12:89-100.
Stavrinides, J., Ma, W., and Guttman, D. S. 2006. Terminal reassortment drives the quantum evolution of type III effectors in bacterial pathogens. PLoS Pathog. 2:e104.

Stecher, G., Tamura, K., and Kumar, S. 2020. Molecular evolutionary genetics analysis (MEGA) for macOS. Mol. Biol. Evol. 37:12371239

Strub, C., Alies, C., Lougarre, A., Ladurantie, C., Czaplicki, J., and Fournier, D. 2004. Mutation of exposed hydrophobic amino acids to arginine to increase protein stability. BMC Biochem. 5:9.

Su, J., Spears, B. J., Kim, S. H., and Gassmann, W. 2018. Constant vigilance: Plant functions guarded by resistance proteins. Plant J. 93 637-650.

Wroblewski, T., Caldwell, K. S., Piskurewicz, U., Cavanaugh, K. A., Xu, H., Kozik, A., Ochoa, O., McHale, L. K., Lahre, K., Jelenska, J., Castillo, J. A., Blumenthal, D., Vinatzer, B. A., Greenberg, J. T., and Michelmore, R. W. 2009. Comparative large-scale analysis of interactions between several crop species and the effector repertoires from multiple pathovars of Pseudomonas and Ralstonia. Plant Physiol. 150: 1733-1749.

Xin, X.-F., and He, S. Y. 2013. Pseudomonas syringae pv. tomato DC3000: A model pathogen for probing disease susceptibility and hormone signaling in plants. Annu. Rev. Phytopathol. 51:473-498. 